\title{
Multispacecraft Observations of Magnetic Clouds and Their Solar Origins between 19 and 23 May 2007
}

\author{
E.K.J. Kilpua · P.C. Liewer • C. Farrugia · J.G. Luhmann • C. Möstl • Y. Li · Y. Liu • \\ B.J. Lynch - C.T. Russell • A. Vourlidas - M.H. Acuna - A.B. Galvin • D. Larson • \\ J.A. Sauvaud
}

Received: 24 June 2008 / Accepted: 25 November 2008 / Published online: 20 December 2008

(C) The Author(s) 2008. This article is published with open access at Springerlink.com

\begin{abstract}
We analyze a series of complex interplanetary events and their solar origins that occurred between 19 and 23 May 2007 using observations by the STEREO and Wind satel-
\end{abstract} Electronic supplementary material The online version of this article
(http://dx.doi.org/10.1007/s11207-008-9300-y) contains supplementary material, which is available to
authorized users.

E.K.J. Kilpua · J.G. Luhmann · Y. Li · Y. Liu · B.J. Lynch · D. Larson

Space Sciences Laboratory, University of California, Berkeley, CA, USA

E.K.J. Kilpua $(\bowtie)$

Department of Physical Sciences, Theoretical Physics Division, University of Helsinki, Helsinki,

Finland

e-mail: emilia.kilpua@ helsinki.fi

P.C. Liewer

Jet Propulsion Laboratory, California Institute of Technology, Pasadena, CA 91109, USA

C. Farrugia - A.B. Galvin

Institute for the Study of Earth, Oceans, and Space, University of New Hampshire, Durham, NH 03824, USA

C. Möstl

Institute of Physics, University of Graz, Universitaetsplatz 5, 8010 Graz, Austria

C. Möstl

Space Research Institute, Austrian Academy of Sciences, Schmiedlstr. 6, 8042 Graz, Austria

C.T. Russell

Institute of Geophysics and Planetary Physics, UCLA, Los Angeles, CA, USA

A. Vourlidas

Solar Physics Branch, Naval Research Laboratory, Washington, DC 20375, USA

M.H. Acuna

NASA/Goddard Space Flight Center, Code 674, Greenbelt, MD 20771, USA

J.A. Sauvaud

CESR/CNRS, 9 avenue du Colonel Roche, Toulouse, 31028, France 
lites. The analyses demonstrate the new opportunities offered by the STEREO multispacecraft configuration for diagnosing the structure of in situ events and relating them to their solar sources. The investigated period was characterized by two high-speed solar wind streams and magnetic clouds observed in the vicinity of the sector boundary. The observing satellites were separated by a longitudinal distance comparable to the typical radial extent of magnetic clouds at $1 \mathrm{AU}$ (fraction of an $\mathrm{AU}$ ), and, indeed, clear differences were evident in the records from these spacecraft. Two partial-halo coronal mass ejections (CMEs) were launched from the same active region less than a day apart, the first on 19 May and the second on 20 May 2007. The clear signatures of the magnetic cloud associated with the first CME were observed by STEREO B and Wind while only STEREO A recorded clear signatures of the magnetic cloud associated with the latter CME. Both magnetic clouds appeared to have interacted strongly with the ambient solar wind and the data showed evidence that they were a part of the coronal streamer belt. Wind and STEREO B also recorded a shocklike disturbance propagating inside a magnetic cloud that compressed the field and plasma at the cloud's trailing portion. The results illustrate how distant multisatellite observations can reveal the complex structure of the extension of the coronal streamer into interplanetary space even during the solar activity minimum.

Keywords Magnetic cloud $\cdot$ Solar wind $\cdot$ Coronal mass ejection $\cdot$ Helmet streamer

\section{Introduction}

During the time of solar activity minimum, the heliosphere is in a relatively simple state and dominated by recurrent high-speed solar-wind streams from large coronal holes bounding the denser and slower flowing plasma. The slow solar-wind component has been interpreted as the interplanetary extension of the bright helmet arcades that form the coronal streamer belt (Gosling et al., 1981). The structure of the coronal streamer belt in interplanetary space is highly variable, featuring activity from quiet, but structured, outflow to intrusions of largescale transients such as coronal mass ejections (CMEs) (e.g., Crooker et al., 1993).

Several studies (e.g., Cane, Richardson, and St. Cyr, 1998; Webb et al., 2000) have linked the front-side full and partial-halo CMEs with specific solar-wind plasma and magnetic-field signatures commonly called interplanetary CMEs (ICMEs) (e.g., Neugebauer and Goldstein, 1997). Magnetic clouds form a substantial subset of ICMEs defined by simple properties (Burlaga et al., 1981): an above-average magnetic field, a large rotation of the magnetic field direction, and a low proton plasma $\beta$. Counterstreaming suprathermal electron events are also frequently observed during magnetic cloud intervals, indicating closed magneticfield configurations (i.e., with field lines still connected to the Sun) (Gosling, Birn, and Hesse, 1995). A large fraction of CMEs arise from the streamer belt (Hundhausen, 1993) and consequently magnetic clouds are often observed in the leading edges of high-speed streams (e.g., Fenrich and Luhmann, 1998) and at sector boundaries (Crooker, Gosling, and Kahler, 1998).

Near the orbit of Earth, magnetic clouds are mesoscale (fraction of an AU) configurations, and therefore to examine the large-scale properties of magnetic clouds, we need observing satellites having separations of this order of magnitude (Mulligan et al., 1999). Magnetic clouds are often modeled as cylindrically symmetric, constant- $\alpha$ force-free configurations (Burlaga, 1988), but it was widely appreciated at the outset that this is but a first-order approximation. Least-squares fits of the data to this model often showed large deviations, most notably in the temporal profile of the magnetic field $(B)$ (Lepping, Jones, 
Figure 1 Locations of Wind (black diamond), STEREO A (red cross), and STEREO B (blue triangle) satellites on 22 May 2007 in the GSE $X Y$-plane. Sketch of (a) MC1 and (b) MC2. The ellipses have been scaled based on the observations at different satellites, and contours from Grad - Shafranov reconstruction (Figure 6) projected in the $X Y$-plane have been overlaid on the ellipses (see text for details).

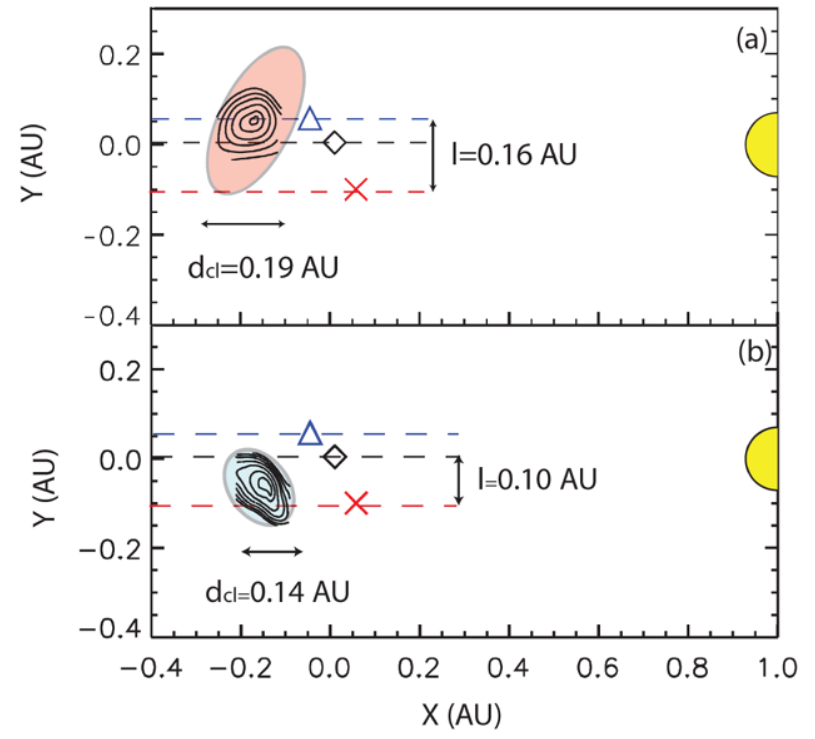

and Burlaga, 1990). Further, the attempts to fit the same cylindrical flux-rope model to distant multipoint cloud observations have suggested distortions that are either inherent to the ejecta or are produced in transit in the structured solar wind (e.g., Mulligan and Russell, 2001; Riley et al., 2003; Liu et al., 2006).

The observations by the Solar Terrestrial Relations Observatory (STEREO) (Kaiser et al., 2007), which was launched in October 2006, offer new opportunities to address important questions related to the large-scale structure of magnetic clouds and the solar wind. STEREO consists of two functionally-identical satellites, one that leads Earth (STEREO A) and one that lags Earth (STEREO B) in its orbit around the Sun with gradually-increasing angular separation. Each spacecraft of the STEREO mission carries suites of remote sensing and in-situ instruments, allowing unprecedented possibilities of relating in-situ and solar observations.

The purpose of this paper is to take advantage of distant multispacecraft observations to analyze the complex solar and interplanetary events that took place on 19-23 May 2007, coinciding with the activity minimum of the solar cycle. At that time Wind and ACE were orbiting around the $\mathrm{L}_{1}$ point and the STEREO satellites were separated by $\approx 9^{\circ}$ (Figure 1 ). All four spacecraft were close to the ecliptic plane. The separation of the STEREO spacecraft was $2418 R_{\mathrm{E}}(\approx 0.10 \mathrm{AU})$ in the radial and $3685 R_{\mathrm{E}}(\approx 0.16 \mathrm{AU})$ in the East-West (longitudinal) directions. Wind and ACE observed almost identical solar-wind conditions (Liu et al., 2008), and we shall use only Wind measurements to describe conditions at $\mathrm{L}_{1}$. Two partial halo CMEs were launched less than a day apart, the first one on 19 May 2007 and the second one on 20 May 2007. We shall argue that the first CME was associated with a magnetic cloud observed at STEREO B and Wind, whereas clear signatures of the second CME were observed only by STEREO A. Both CMEs were associated with a small B-class flare and a filament eruption in active region (AR) 10956 as well as coronal dimming.

We start by describing in-situ observations by STEREO B and Wind followed by an analysis of STEREO A data in Section 2. In Section 3 we discuss the associated solar events and how they connect with the interplanetary observations. Finally, in Section 4, we discuss the observations, and in Section 5 we summarize the results. 

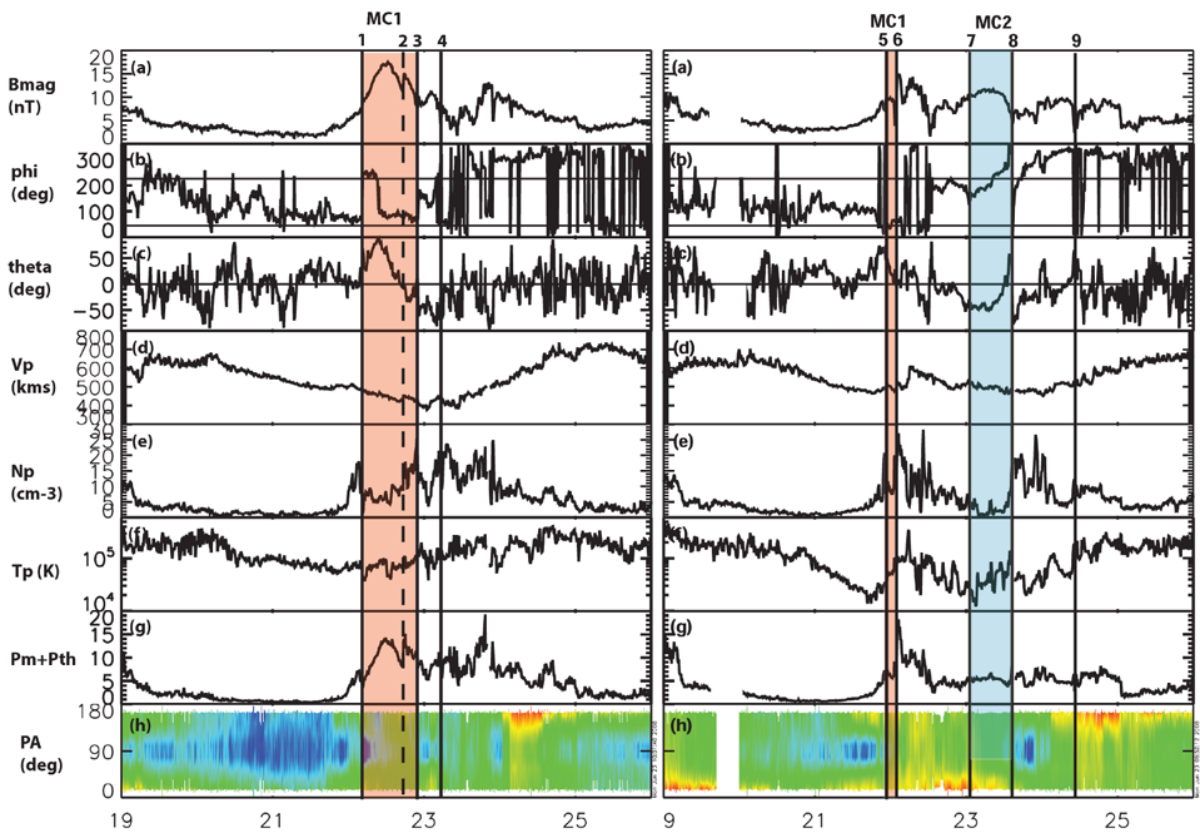

Figure 2 STEREO B (left) and STEREO A (right) measurements for a seven-day interval between 19 and 26 May 2007: (a) magnetic-field magnitude, (b) magnetic-field azimuth angle $(\phi)$ in GSE coordinates, (c) magnetic-field latitude angle $(\theta)$ in GSE coordinates, (d) solar-wind speed, (e) solar-wind density, (f) solar-wind temperature, and $(\mathrm{g})$ total pressure (thermal and magnetic) in units of $10^{-11} \mathrm{~Pa}$. A constant electron temperature of $130000 \mathrm{~K}$ and an $\alpha$-to-proton ratio of $4 \%$ were assumed. Panel (h) shows the pitch-angle spectrogram of 246-eV electrons. Regions of different colors and vertical lines indicate periods of interest that will be discussed in the text. The horizontal lines mark the magnetic-field azimuth angles $45^{\circ}$ and $225^{\circ}$. "MC1" refers to a magnetic cloud observed clearly by STEREO B and Wind and "MC2" refers to a magnetic cloud observed by STEREO A (see Table 1 and the text for details).

\section{Interplanetary Observations}

During mid-May 2007 the Wind and STEREO satellites observed a complex sequence of events. Figure 2 shows a seven-day period of solar wind plasma and magnetic field measurements acquired by the STEREO satellites. As we shall see, the Wind satellite located $\approx 3^{\circ}$ from STEREO B and almost $6^{\circ}$ from STEREO A observed solar-wind conditions roughly similar to those at STEREO B.

As seen from Figure 2d, during the investigated period two high-speed solar wind streams swept past the satellites. These high-speed streams were emanating from two-large coronal holes visible in the STEREO A/Extreme UltraViolet Imager (EUVI) image at $195 \AA$ bandpass (Figure 3). The magnetic-field azimuth angle $(\phi)$ in GSE coordinates (Figure 2b) indicates that there was a structured passage from an away $\left(\phi\right.$ within $45^{\circ}-225^{\circ} ; 135^{\circ}$ on average) to a toward $\left(\phi=315^{\circ}\right.$ on average) magnetic sector. The pitch-angle spectrograms of 246-eV electrons (Figure $2 \mathrm{~g}$ ) show the corresponding change in the dominant heat flux direction early on 24 May, confirming that these transitions include true sector boundaries. Figure 4 shows the synoptic ecliptic-plane field plot by the Global Oscillation Network Group (GONG) coronal-field source surface model. The polarity of the solar magnetic field changes each solar cycle and, as illustrated by Figure 4, during solar cycle 23 positivepolarity fields (away sector) were concentrated on the southern hemisphere and negative- 


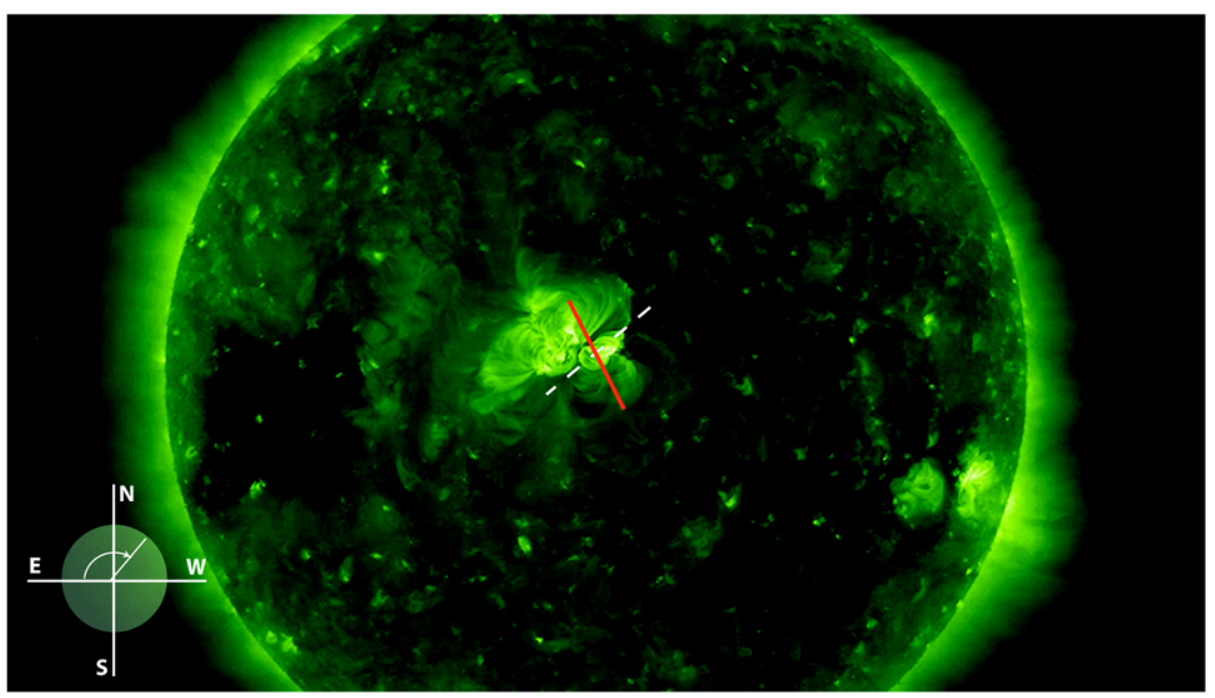

Figure 3 EUVI image in the $195 \AA$ bandpass by STEREO A/SECCHI/EUVI on 19 May 2007 at 14:42 UT. The image features the solar active region 10956 surrounded by two large coronal holes. The circle in the lower left corner illustrates the coordinate system used to determine the orientation angles (Yurchyshyn, 2008). The white dashed line shows the approximate orientation of post-eruptive arcades $\left(140^{\circ}\right)$ the red line indicates the orientation angle of MC1 (see Section 3).

polarity fields (toward sector) on the northern hemisphere. Figure 4 further demonstrates that the observed interplanetary polarity patterns match those predicted by the GONG model at the period investigated. Note that in mid and early April source-surface model predictions do not match the observed polarity pattern.

The colored areas and vertical lines in Figure 2 mark the interesting structures identified between the high-speed streams that will be analyzed in detail. They straddle the region between the trailing end of one stream and the leading edge of the following stream. The most prominent feature in the data is an interval of high and smooth magnetic field accompanied by organized rotation of the magnetic field at STEREO B on 22 May.

\subsection{STEREO B and Wind Observations}

In Figure 5 we compare the details of the magnetic field and solar-wind plasma observations at Wind and STEREO B spanning the pink area of Figure 2. In Figure 5 the Wind data have been shifted to the time at STEREO B using a 6.07-hour convection time ( $\left.t_{\text {conv, WB }}\right)$ as estimated by aligning a sharp directional change of the magnetic field and drop in the proton $\beta$ (vertical line 1). After this, the defining magnetic-cloud signatures were observed: enhanced and smooth magnetic field, organized rotation of the magnetic field, and depressed proton $\beta$. In addition, the pitch-angle spectrogram of 246-eV suprathermal electrons (Figure $5 \mathrm{~h}$ ) shows heat-flux electrons to be counterstreaming along the magnetic field. The occasional dropouts of the bidirectional electron flux within the magnetic cloud and the start of the counterstreaming hours before the leading edge are features reported in earlier studies (Shodhan et al., 2000). We defined the end boundary of this magnetic cloud at the time indicated by line 3 where the main magnetic field rotation ended. Table 1 lists several key parameters of this magnetic cloud, called from now on "MC1." The average solar-wind 


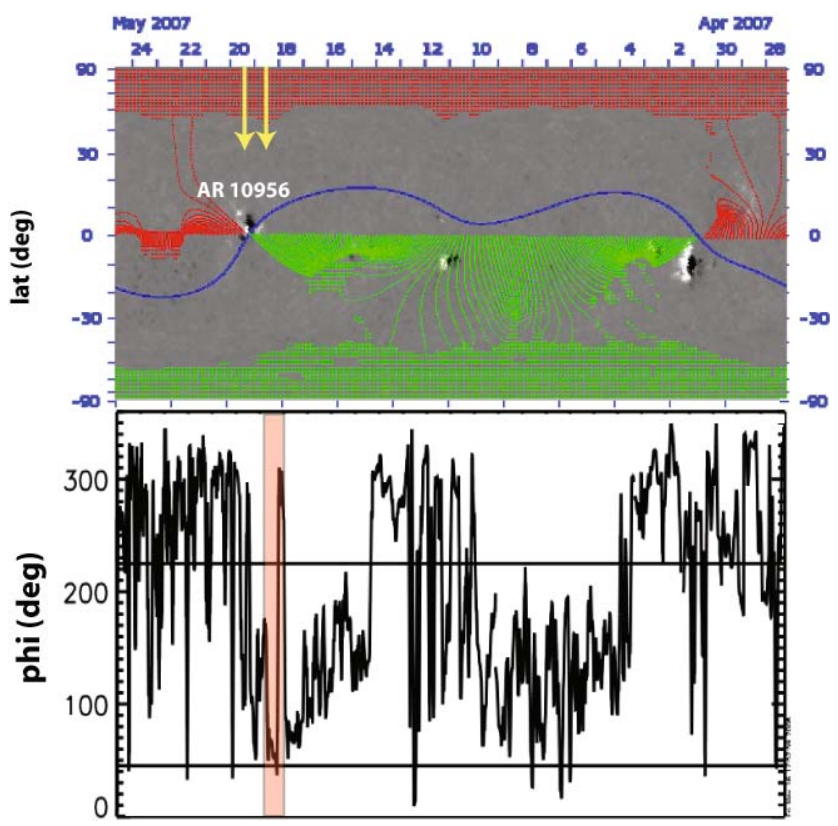

Figure 4 (a) The GONG synoptic ecliptic-plane field plot for the Carrington rotation 2056 (Web site plot courtesy of Gordon Petrie, NSO). The model shows the field lines that are open to the heliosphere at the ecliptic plane. The thick blue line shows the neutral line on the source surface (fixed at 2.5 solar radii) of a potential-field source-surface model. The green dots and lines indicate positive polarity coronal holes and the open ecliptic field lines, respectively; the red dots and lines indicate negative polarity. The yellow arrows mark the solar launch times of the CMEs thought to be sources of the observed magnetic clouds. (b) Magnetic azimuth angle $(\phi)$ in GSE coordinates at Wind using the time shift of 3.5 days from the Sun to the Wind. The pink region marks the interval of the $\mathrm{MC} 1$ at Wind.

speed and the duration of magnetic cloud passage at STEREO B yield an estimated magnetic cloud scale size in the radial direction, $\left(d_{\mathrm{CL}}\right)$ of $\approx 0.19 \mathrm{AU}$, a fairly typical value and one comparable to the spacecraft separations. The generally decreasing bulk speed profile indicates a radial expansion of the cloud. Dividing the radial separation distance between the spacecraft by $t_{\text {conv, WB }}$ yields a radial propagation speed of $486 \mathrm{~km} \mathrm{~s}^{-1}$, which matches very well the leading edge speed of the magnetic cloud (Table 1).

Line 2a in Figure 5 marks a sharp increase in the magnetic field strength and solar-wind speed as well as in the plasma density at 17:30 UT on 22 May at STEREO B. The conservation of momentum flux yields a shock speed of $493 \mathrm{~km} \mathrm{~s}^{-1}$, and the coplanarity theorem gives the shock normal $\mathbf{N}_{\mathrm{SH}}=(0.858,-0.497,-0.131)$ in GSE. Using the calculated shock speed and the separation of Wind and STEREO B along the shock normal of $1137 R_{\mathrm{E}}$ yields an estimated travel time between these satellites of 4.1 hours, suggesting that the shock should pass the location of Wind on 22 May at 13:24 UT. Wind recorded a clear, but more gradual increase in the plasma density and temperature (not shown) at 13:45 UT on 22 May (line 2b), fairly consistent with the estimated shock arrival time. However, no increase in the solar-wind speed or magnetic field strength was observed at Wind.

The last row of Table 1 gives the axis orientation of the MC1 derived from the reconstruction of the magnetic cloud by solving numerically the Grad-Shafranov equation (Hu and Sonnerup, 2002) on the STEREO B data. A Grad-Shafranov reconstruction (GSR) is applicable to structures with an invariant direction $(z)$ and has been successfully used to study 


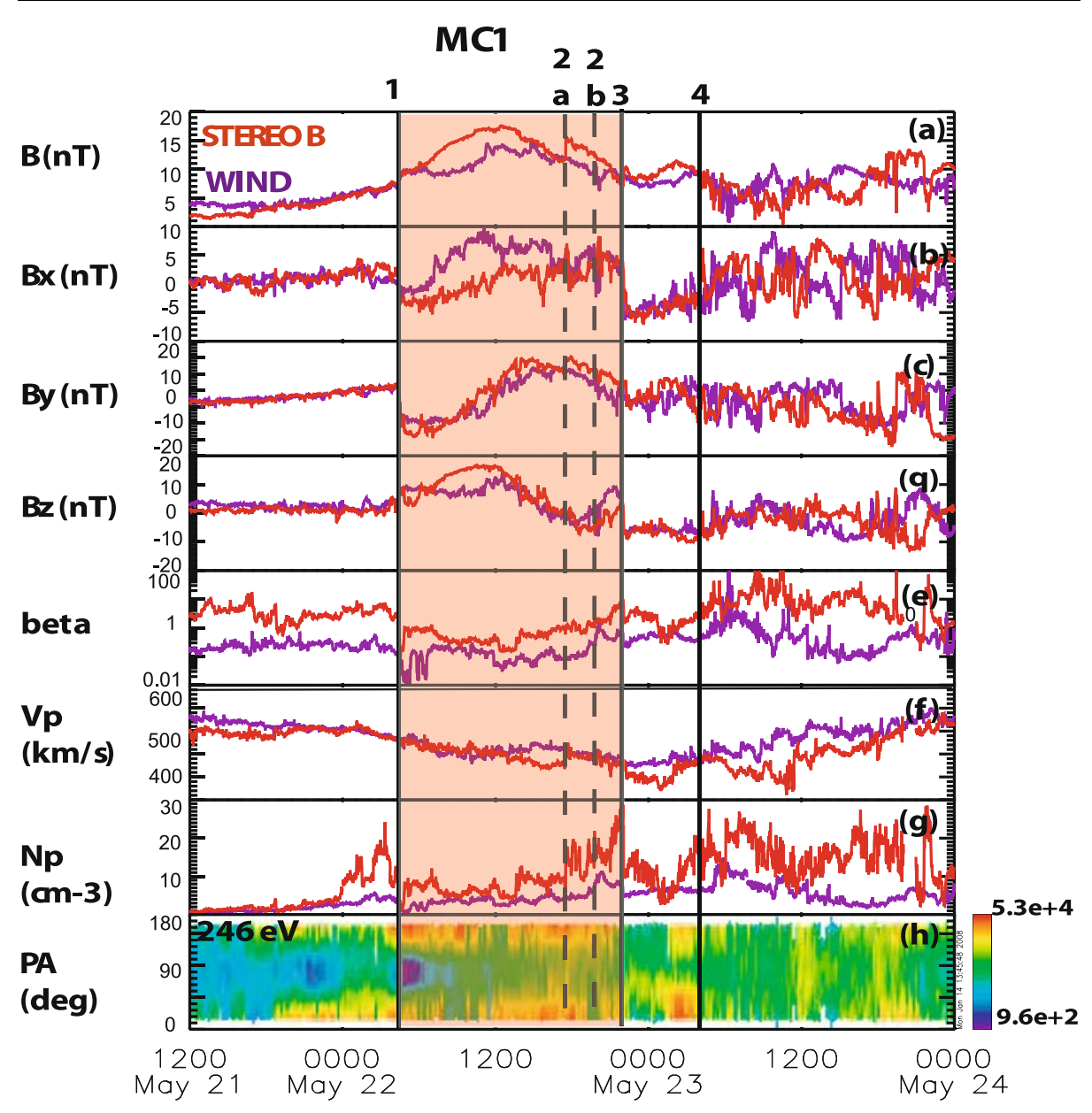

Figure 5 Comparison of Wind and STEREO B observations from 21 May at 12 UT to 24 May at 00 UT 2007: (a) magnetic-field magnitude, (b) - (d) magnetic-field GSE components, (e) the proton $\beta$, (f) solar-wind speed, (g) solar wind density, and (h) the pitch-angle spectrogram of 246-eV electrons at STEREO B. Vertical lines mark the times of interest discussed in the text.

MCs modeled as magnetic flux ropes. Here, the plasma velocity components were not available, so we assume a radial propagation: $V_{X}=-|\mathbf{V}|$ and $V_{Y}=V_{Z}=0$. The cross section of the MC is illustrated in Figure $6 a$, where the contour lines give the transverse magnetic field lines and the axial field strength is shown color-coded, maximizing at the white dot. For a successful reconstruction, the transverse pressure $P_{\mathrm{t}}(A)=p+B_{z}^{2} / 2 \mu_{0}$, where $A$ is the vector potential and $p$ the plasma pressure, has to be single-valued. With a small fitting residue (Hu et al., 2004) of $R_{\mathrm{f}}=0.05$ this condition is well satisfied. The axis orientation in the GSR technique is determined by finding iteratively the direction for which the data plot of $P_{\mathrm{t}}$ versus $A$ displays minimal scatter. The result suggests that at STEREO B MC1 had an axis that was highly inclined to the solar ecliptic plane $\left(\theta_{\mathrm{CL}} \approx 63^{\circ}\right)$. Note that GSR analysis confines the MC1 approximately between lines 1 and 2, but based on visual inspection the end boundary of the MC1 was placed about 4.5 hours later. Figure $7 \mathrm{a}$ also shows $3 \mathrm{D}$ helical field lines based on the GS maps viewed in the GSE $X Y$-plane. It is seen from the figure 
Table 1 Summary of the observations during a magnetic cloud observed at the locations of Wind and STEREO B (MC1) and during a magnetic cloud observed at STEREO A (MC2). Rows give (1) spacecraft radial distance from the Sun, (2) the GSE separation vectors of Wind and STEREO A from STEREO B, $(3,4)$ the interval of the magnetic cloud, (5) leading edge speed/average solar-wind velocity, (6) radial scale size of the cloud, (7) maximum value of the magnetic field, (8) time of the shocklike disturbance at STEREO B and the time of the pressure increase at Wind, and (9) axis orientation from the GSR analysis (see Hu et al., 2004, for the determination of the error bars).

\begin{tabular}{lllll}
\hline & & STEREO B $(\mathrm{MC} 1)$ & Wind $(\mathrm{MC} 1)$ & STEREO A (MC2) \\
\hline 1 & $d(\mathrm{AU})$ & 1.06 & 0.99 & 0.96 \\
2 & $(\Delta X, \Delta Y, \Delta Z)$ & - & $(0.055,0.052,0.0060)$ & $(0.10,0.16,0.0065)$ \\
3 & start (UT) & $05 / 2204: 25$ & $05 / 2122: 45$ & $05 / 2300: 56$ \\
4 & end (UT) & $05 / 2222: 05$ & $05 / 2215: 55$ & $05 / 2312: 24$ \\
5 & $V\left(\mathrm{~km} \mathrm{~s}^{-1}\right)$ & $481.1 / 447.4$ & $482.6 / 456.3$ & $535.0 / 497.2$ \\
6 & $d_{\mathrm{CL}}$ & 0.19 & 0.19 & 0.14 \\
7 & $B_{\max }(\mathrm{nT})$ & 17.6 & 14.8 & 11.8 \\
8 & $T_{\mathrm{S}}$ & $22 \mathrm{May} 17: 30$ & 22 May $13: 42$ & - \\
9 & $\mathrm{GS}\left(\phi_{\mathrm{CL}}, \theta_{\mathrm{CL}}\right)$ & $\left(63^{\circ} \pm 8^{\circ}, 76^{\circ} \pm 8^{\circ}\right)$ & - & $\left(-58^{\circ} \pm 4^{\circ}, 220^{\circ} \pm 4^{\circ}\right)$ \\
\hline
\end{tabular}

that $\mathrm{MC} 1$ crosses eastward of Earth and that the axial field (red arrow along the axis) points northward.

We now determine the toroidal and poloidal magnetic fluxes associated with MC1 following Qiu et al. (2007). We set an outer boundary at $A_{\mathrm{b}}=26 \mathrm{Tm}$, corresponding to the white contour line in Figure $6 \mathrm{a}$. At the center of the cloud, $A_{\mathrm{m}}=81 \mathrm{Tm}$. We thus find a toroidal flux $\Phi_{\mathrm{t}}=\iint B_{z} \mathrm{~d} x \mathrm{~d} y$ of $0.21 \times 10^{21} \mathrm{Mx}$. The poloidal flux is $\Phi_{\mathrm{p}}=\left|A_{\mathrm{m}}-A_{\mathrm{b}}\right| L$, and thus $0.82 \times 10^{21} \mathrm{Mx} \mathrm{AU}^{-1}$. We note that both should be seen as lower limits, as it is not possible to reconstruct field lines of the cloud beyond the discontinuity. Figure 6 also indicates right-handed chirality for MC1.

The general behavior of the magnetic-field components during the passage of $\mathrm{MC} 1$ are roughly similar at Wind and STEREO B, but the magnetic-field profile was clearly more symmetric, the peak magnetic-field magnitude higher, and the magnetic-field directional changes more organized at STEREO B. GSR analysis suggests that STEREO B crossed $\mathrm{MC} 1$ almost centrally, with the impact parameter (i.e., the closest crossing distance of the spacecraft from the magnetic cloud axis) being $0.000_{-0.006}^{+0.003} \mathrm{AU}$ (with errors derived from the estimated error in the axis orientation). The invariant axis cannot be defined clearly for the Wind data, presumably because of the large estimated impact parameter $\left(\approx 0.3 d_{\mathrm{CL}}\right)$ and the probable distortion of the magnetic field owing to the interaction with the leading edge of the trailing high-speed stream. [See Liu et al. (2008) for another application of the GSR method to this magnetic cloud, with similar conclusions regarding the inferred axis orientation.]

In Figures 1a and 1b we give a schematic representation of the geometry for the two magnetic clouds, using satellite observations made roughly in the ecliptic plane and the two cloud orientations derived from the GSR analysis. The ellipse in Figure 1a illustrates the cross section of MC1 in the GSE $X Y$-plane, where the observations are made. The ellipse has been scaled by using the observed cloud scale size in the radial direction (0.19 AU) and the impact parameter from GSR analysis. It was assumed that STEREO A (symbol X) encountered just the flanks of MC1 (see the discussion in Section 2.2). The orientation of the ellipse in the $X Y$-plane is as deduced from the GSR-inferred axis orientation. The major axis of the resulting ellipse is $0.36 \mathrm{AU}$ and the minor axis is $0.16 \mathrm{AU}$. In Figure 1 we have also overlaid the contours from the GSR analysis (i.e., transverse magnetic-field lines) projected 
(a)

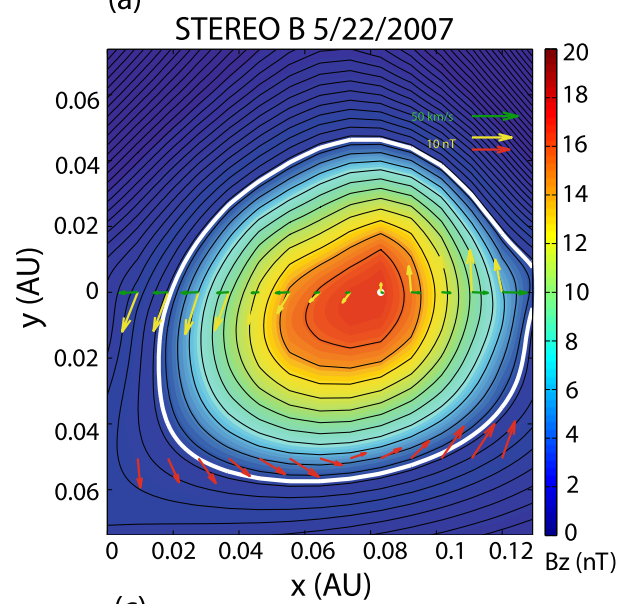

(c)

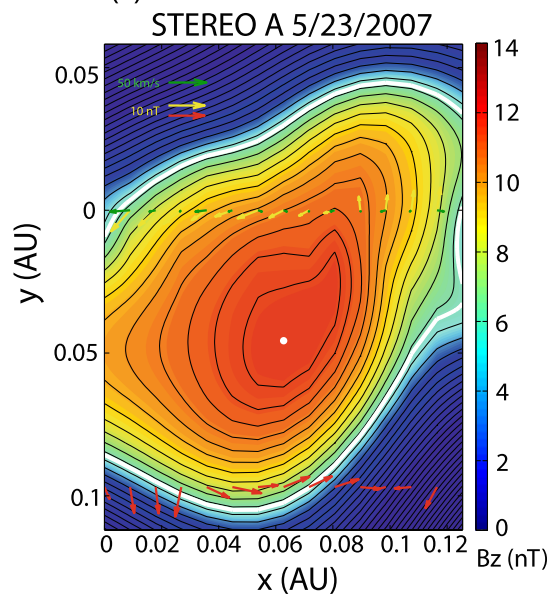

(b)

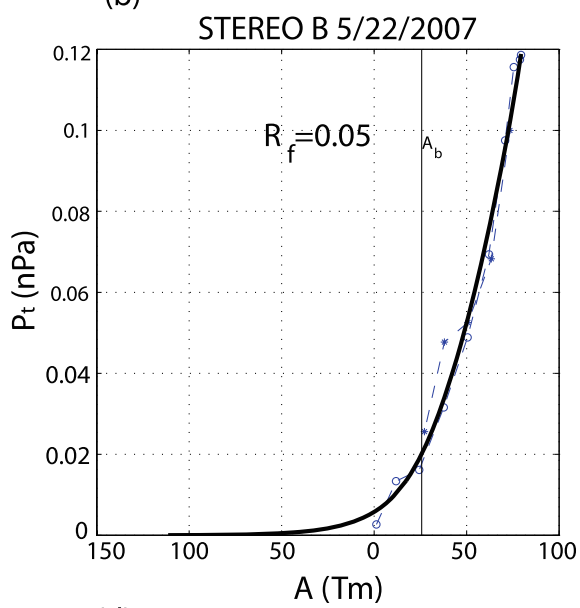

(d)

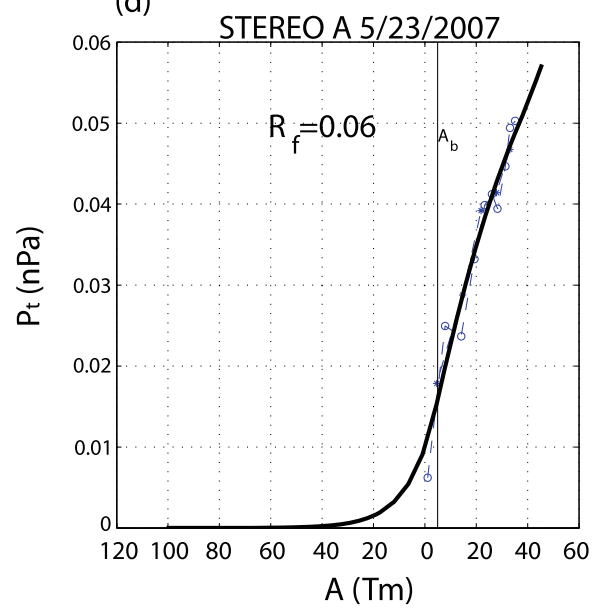

Figure 6 Reconstruction of (a) the MC1 and (c) the MC2 in the plane perpendicular to the magnetic cloud axis from STEREO B and STEREO A measurements, respectively, using the Grad - Shafranov method. White dot shows the magnetic-field maximum. The yellow arrows show the magnetic-field direction and magnitude along the STEREO B (a) and STEREO A (c) trajectory while the red arrows indicate the magnetic-field measurements at Wind assuming that the cloud propagated to Wind at the constant Hoffman-Teller frame velocity. Plots of the measured transverse pressure $\left(P_{\mathrm{t}}\right)$ versus the vector potential $(A)$ along $Y=0$ for the reconstruction interval for (a) MC1 and for (b) MC2. The black curve shows the fitted $P_{\mathrm{t}}$; circles (stars) are the data along the STEREO B inbound (outbound) path.

onto the Ecliptic. It is evident that the contour plot is much smaller than the extent of MC1 estimated from observations for the following reasons: $i$ ) The shocklike discontinuity does not allow GS reconstruction of field lines after the time of its appearance and $i$ ) the extent of the GS maps transverse to the MCs direction of motion is constructed by numerical errors (e.g., Hu and Sonnerup, 2002).

As seen from Figure 5, a small (0.08 AU) magnetic cloudlike region follows the end boundary of the MC1, delimited by lines 3 and 4 . Counterstreaming heat flux electrons were observed at both Wind and STEREO B during this structure, but the magnetic-field and 

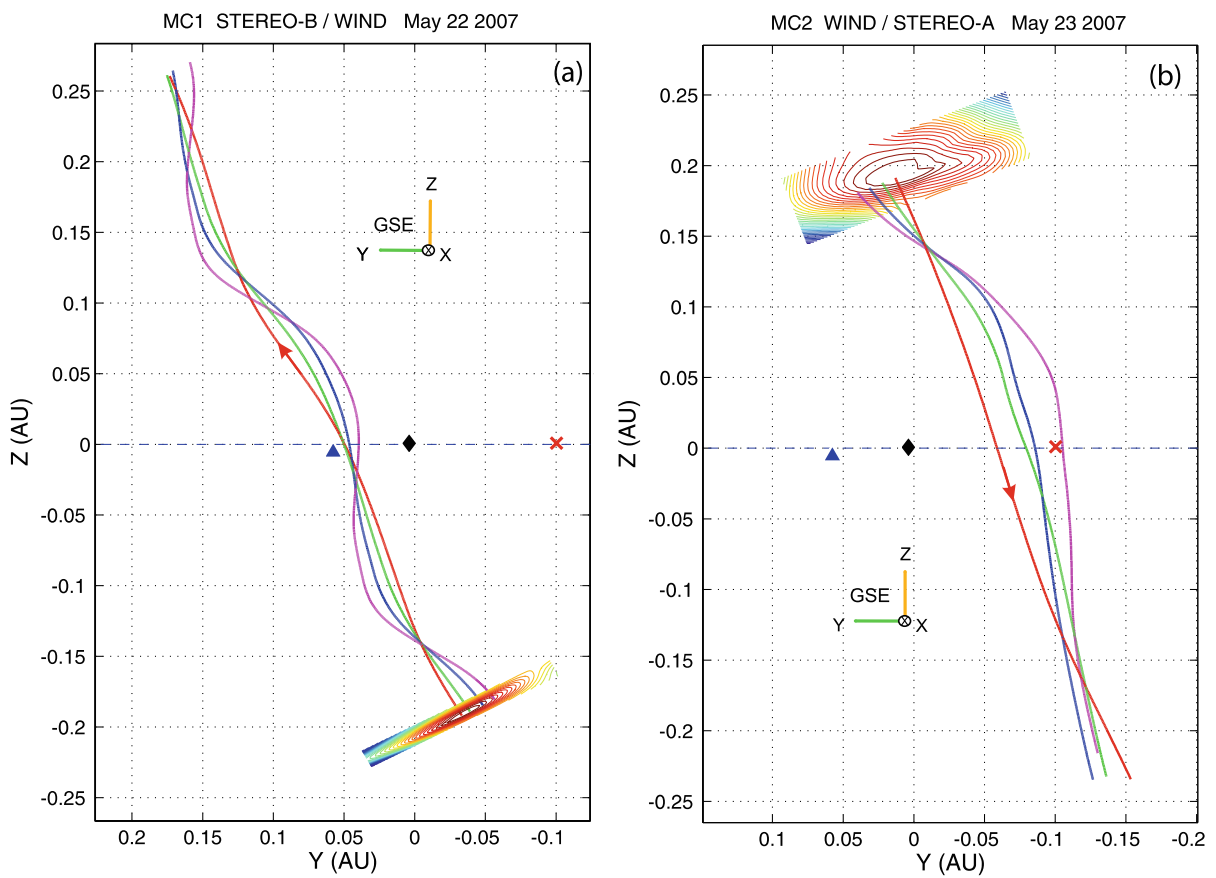

Figure 7 Representation of the GS maps (Figure 6) as 3D helical field lines, viewed in the GSE $X Z$-plane looking toward the Sun (GSE $+X)$ for (left) MC1 and (right) MC2. The spacecraft are marked as in Figure 1; the coordinate system is centered on Earth. Arrows along red field lines (MC axes) indicate the axial field direction. Invariance for $0.5 \mathrm{AU}$ along the $\mathrm{MC}$ axes was assumed.

plasma characteristics were more pronounced at the location of STEREO B. Disorganized electron heat flux signatures and fluctuating magnetic-field azimuth angle characterized the region between the end boundary of the above-described small magnetic cloud and the reversal of electron heat flux on 24 May (Figure 2).

\subsection{STEREO A Observations}

Figures 2, 5, and 8 demonstrate that STEREO A recorded distinctly different plasma and magnetic-field characteristics than those observed by STEREO B and Wind. GSR analysis of MC1 suggests that STEREO A was located generally outside MC1. However, the region between lines 5 and 6 in Figures 2 and 8 shows an increased total pressure and roughly similar trend in the magnetic-field components to those seen at STEREO B and Wind during the passage of MC1. It is thus possible that STEREO A encountered the flanks of the $\mathrm{MC} 1$ (but note that, owing to strong interaction with the ambient solar wind, the end time of $\mathrm{MC1}$ at STEREO A is not clear). Timing arguments would also support this interpretation: The time difference between the observations of the MC1 leading edge at STEREO B and the structure indicated by line 5 at STEREO A was 9.4 hours. Dividing the radial separation distance from STEREO A, and B by this convection time yields a radial propagation speed of $488 \mathrm{~km} \mathrm{~s}^{-1}$, which matches well the speed at STEREO A at the time indicated by line $6\left(\approx 474 \mathrm{~km} \mathrm{~s}^{-1}\right)$. We therefore conclude that MC1 was encountered by STEREO B and Wind whereas STEREO A probably made only a glancing encounter with it, as suggested in Figure 1. 


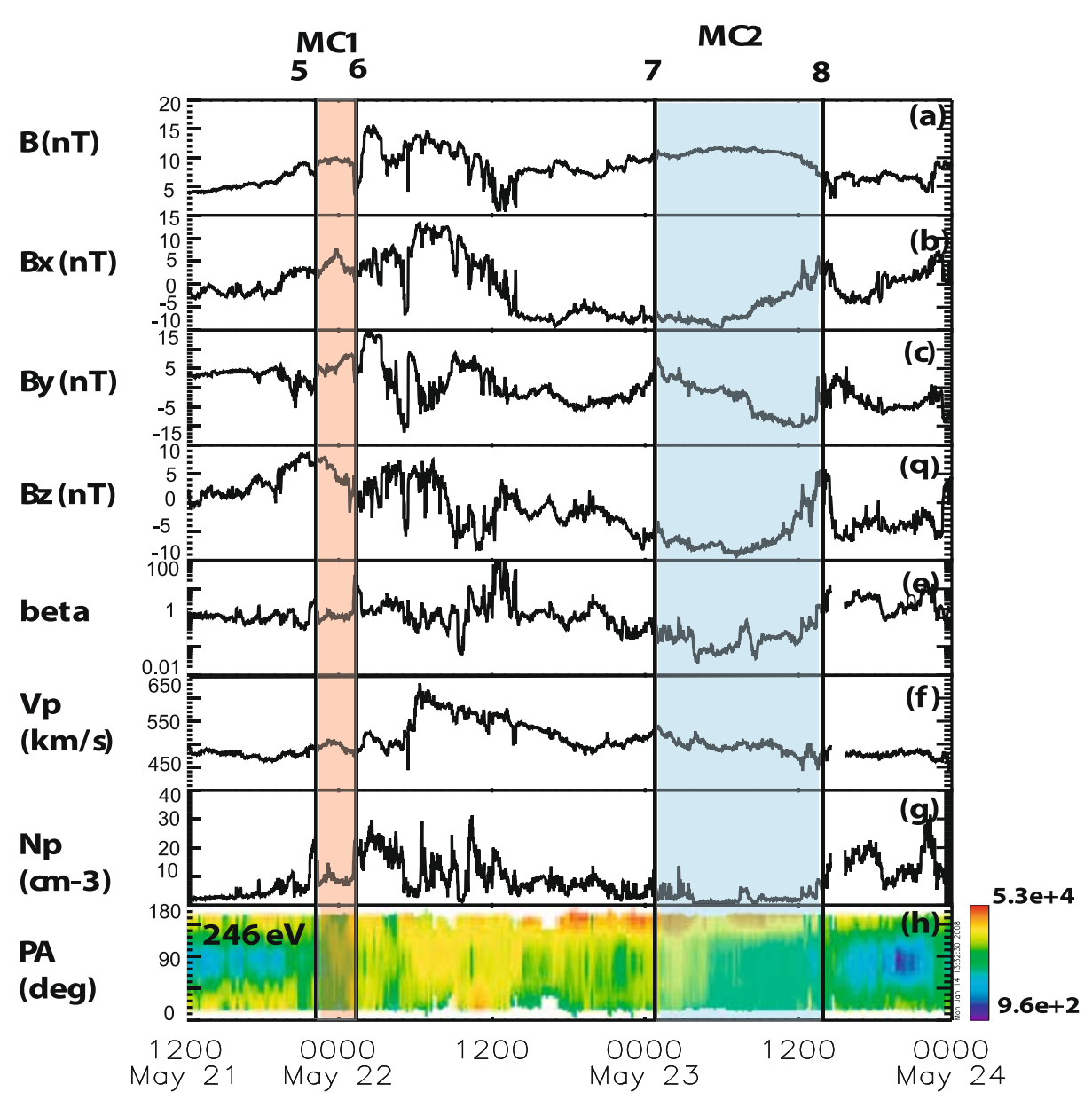

Figure 8 STEREO A observations from 21 May at 12 UT to 24 May at 00 UT 2007. (a) magnetic-field magnitude, (b) - (d) magnetic-field GSE components, (e) the proton $\beta$, (f) solar-wind speed, (g) solar wind density, and (h) the pitch-angle spectrogram of 246-eV electrons at STEREO B. Vertical lines mark the times of interest discussed in the text.

On 23 May 2007 STEREO A observed a magnetic cloud, delimited by lines 7 and 8 in Figures 2 and 8, called from now on "MC2". The observations during MC2 are summarized in Table 1. We do not believe that MC2 and MC1 refer to the same large-scale transient. First, it is seen from Figures 5 and 8 that the variations in the components of the magnetic field were, roughly, in the opposite sense to those seen during MC1's passage at STEREO B and at Wind. The axis of MC2 deduced from GSR analysis (Table 1) is also roughly orientated opposite to the axis of MC1. Second, although the observing spacecraft STEREO A was located closest to the Sun, the leading edge of MC2 arrived at STEREO A (0.96 AU) about a day later than the leading edge of MC1 was observed at STEREO B (1.06 AU) and at Wind $\left(\mathrm{L}_{1}\right)$. And third, there are no indications of counterstreaming electrons during $\mathrm{MC} 2$, implying that magnetic field lines were open (i.e., connected to the Sun only at one end). (Recall that we inferred closed field lines for MC1.) The estimated scale size of MC2 in the radial direction is about $\approx 0.14 \mathrm{AU}$ (i.e., somewhat smaller than $\mathrm{MC} 1$ ). 
The GSR technique was also successful for MC2, with the fitting residue of $R_{\mathrm{f}}=0.06$ (Figure 6). The impact parameter was $0.045 \mathrm{AU}$ for STEREO A $\left(0.32 d_{\mathrm{CL}}\right)$. As shown by the magnetic-field-line map in Figure 6c, the axis of MC2 lies between STEREO A and Wind, and it is seen that Wind should see a small interval of MC2. The small magnetic cloudlike region in Wind data, delimited by lines 3 and 4 and discussed in Section 2.1, is not likely to represent the flanks of MC2: The structure was seen too early on Wind (from 22 May at 16:55 UT to 23:55 UT) and, furthermore, STEREO B also observed this structure (even more pronounced) whereas the results of GSR analysis suggest that MC2 does not extend to the location of STEREO B. The Wind magnetic-field measurements plotted in Figure $6 \mathrm{c}$ (red arrows) show that the rotation agrees reasonably well with the reconstructed map between 0.035 and $0.08 \mathrm{AU}$, which corresponds to the time interval on 23 May from 09:50 UT to 13:30 UT. Figure $7 \mathrm{~b}$ illustrates that MC2 crosses westward of Earth and the axial field points southward. In addition, it is seen from the figure that the magnetic field lines in MC1 are clearly more strongly wound than in $\mathrm{MC} 2$.

For MC2 we find a toroidal flux of $0.33 \times 10^{21} \mathrm{Mx}$ and a poloidal flux of $0.68 \times 10^{21}$

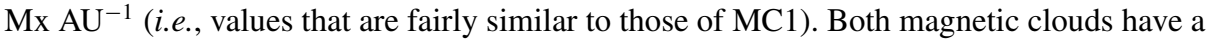
right-handed chirality.

The cross section of MC2 is given in Figure 1b if one assumes that Wind just encountered the flanks of the cloud. Similarly to Figure 1a, the ellipse has been tilted and scaled based on both the observations and the results of GSR analysis. For MC2 the major axis of the ellipse is $0.15 \mathrm{AU}$ and the minor axis is $0.13 \mathrm{AU}$. Comparison of Figures 1a and 1b shows that the MC2 projected cross section is smaller and also more circular than the projected cross section of MC1. Also, for MC2 the overlaid GS contours correspond much better to the scale size estimated from observations than for MC1. Presumably, the GSR technique works better for MC2, because there is no shock as for MC1, so we are able to use the full data interval at STEREO A.

As demonstrated by Figure 2, at STEREO A the change of the magnetic-field azimuth angle $(\phi)$ from an away to a toward sector was gradual and part of the large-scale magnetic field rotation that continued well beyond the end boundary of MC2, until 24 May at 09:50 UT, as marked by line 9 in Figure 2. This region, having a radial extent of $0.23 \mathrm{AU}$, was characterized by a strong depletion of the heat flux electrons at $90^{\circ}$ pitch angle (Gosling, Skoug, and Feldman, 2001).

\section{Solar Observations}

The available remote-sensing data allow us to determine the most likely solar sources for the two observed magnetic clouds and their surrounding structures.

Solar activity during May 2007 was concentrated in active region 10956 (Figure 3), located $\approx 3^{\circ}$ North of the solar Equator. This region produced several small flares (22 GOES $\mathrm{B}$ class and $2 \mathrm{C}$ class) and a number of CMEs when it rotated across the disk between 14 and 22 May.

Although it is known that CME speeds can change substantially in transit through corona and heliosphere, a first cut of source identification can be made by assuming a steady propagation speed. For MC1 a constant extrapolation of the MC1 front speed (482 km s${ }^{-1}$ ) back to the Sun gives a start time about 3.6 days earlier, roughly 12 UT on 18 May. The LASCO CME catalog (Yashiro et al., 2004) shows no significant CMEs on 18 May; only two very slow $\left(V<200 \mathrm{~km} \mathrm{~s}^{-1}\right)$ poor events were reported. On 19 May, the catalog lists two CMEs that occurred very close together (in time and position angle). The faster CME (LASCO catalog linear fit speed $958 \mathrm{~km} \mathrm{~s}^{-1}$ ) appears in LASCO/C2 at 13:24 UT (position angle of $270^{\circ}$; 


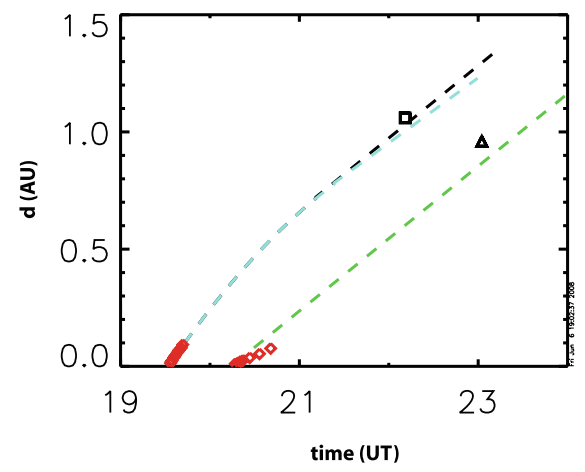

Figure 9 The LASCO/C2 and C3 height - time data (red diamonds) for the fast 19 May CME and the 20 May CME as well as the magnetic-cloud arrival data (square: MC1 at STEREO B; triangle: MC2 at STEREO A); also shown is the empirical prediction for the height - time curve from Gopalswamy et al. (2001) using the measured speeds and transit times. The black curve shows the prediction curve when the MC1 leading edge speed was used and the blue curve shows that when the MC2 leading edge speed was used with the 19 May fast CME initial speed. The green curve shows the prediction curve when the MC2 leading-edge speed and the 20 May CME initial speed were used.

see supplementary online material: movie 1; http://dx.doi.org/10.1007/s11207-008-9300-y) and the slow one (linear fit speed $294 \mathrm{~km} \mathrm{~s}^{-1}$ ) at 13:48 UT (position angle of $310^{\circ}$ ). If this fast CME is the source of MC1, then the average transit speed was about $700 \mathrm{~km} \mathrm{~s}^{-1}$, in between the observed speeds at the Sun $\left(958 \mathrm{~km} \mathrm{~s}^{-1}\right)$ and at $1 \mathrm{AU}\left(482 \mathrm{~km} \mathrm{~s}^{-1}\right)$, supporting the identification. Two other "poor," slow $\left(V<300 \mathrm{~km} \mathrm{~s}^{-1}\right)$ LASCO catalog CMEs on 19 May (at 18:12 UT and 21:48 UT) are not considered to be likely sources. Such small events with no associated other solar signatures would not be expected to produce the large signatures seen in the in-situ data.

The slow 19 May CME at 13:48 UT, given its closeness in time and location to the fast CME at 13:24 UT, may be part of the same CME event, and, in fact, it is difficult to separate these two CMEs in LASCO/C2 and STEREO/COR1. The measured slow speed may result from the plane-of-sky projection of the Earthward-directed CME (Leblanc et al., 2001). As described by Li et al. (2008), AR 10956 had a complex magnetic configuration with multiple neutral lines and highly nonpotential coronal structure, which indeed could produce a complex CME.

For MC2 (leading edge speed of $535 \mathrm{~km} \mathrm{~s}^{-1}$ ), a constant-speed extrapolation gives an estimated start time early on 20 May. At 06:48 UT on 20 May a partial-halo CME was observed (position angle of $201^{\circ}$ ) with a linear fit speed of $275 \mathrm{~km} \mathrm{~s}^{-1}$ (see supplementary online material: movie 2; http://dx.doi.org/10.1007/s11207-008-9300-y). There are no indications of other major events in the suitable time window. It should be noted that owing to the projection effects the true radial speed of a CME might differ significantly from the projected speed (e.g., Plunkett et al., 1998; Leblanc et al., 2001). In fact, an analysis of the speed of this CME, using stereoscopic techniques on COR1 data, gives a de-projected speed of $545 \mathrm{~km} \mathrm{~s}^{-1}$, very close to the observed leading-edge speed of MC2 (Mierla et al., 2008).

More accurate approximations of CME transit times can be made by applying existing models for CMEs propagation through interplanetary space: Figure 9 gives the propagation curves for CMEs leaving the Sun on 19 May at 13:24 UT and on 20 May at 06:48 UT based on the fit to the empirical formula of Gopalswamy et al. (2001), in which the deceleration is a simple function of the initial speed and the leading edge speed at $1 \mathrm{AU}$. The black curve in Figure 8 is obtained by using the initial speed of the fast 19 May CME $\left(958 \mathrm{~km} \mathrm{~s}^{-1}\right)$ and 
Figure $10 \mathrm{H} \alpha$ images from Kanzelhoehe Solar Observatory (a) on 19 May 2007 at 05:19 UT and (b) on 20 May 2007 at 06:42 UT.

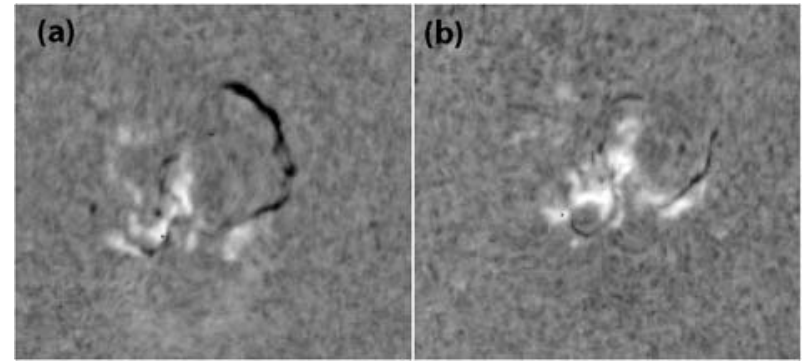

the leading edge speed of MC1; for the blue curve the MC2 leading edge speed is used. The green curve is obtained by using the initial speed of the 20 May CME $\left(545 \mathrm{~km} \mathrm{~s}^{-1}\right)$ and the leading edge speed of MC2. Also shown are the LASCO/C2 and C3 height-time data for the fast 19 May CME and the magnetic clouds arrival data at the STEREO satellites. It can be seen from Figure 9 that STEREO B observed the leading edge just 4.2 hours later than the prediction for the fast 19 May CME whereas STEREO A observed MC2 about a day later. Thus it is unlikely that the fast CME on 19 May is the cause of MC2; the identification of MC2 with the 20 May CME seems much more reasonable.

Both the 19 May and 20 May CMEs were associated with distinct solar surface activity. On 19 May 2007 AR 10956 had just passed the Sun's central meridian when an eruption of a large, curved filament (Figure 10) was seen in STEREO/EUVI 304 and $171 \AA$ data beginning at 12:51 UT. The filament eruption was accompanied by a B9.5class flare that started at 12:48 UT and peaked at 13:00 UT, shortly before the appearances of the CMEs in the LASCO coronagraphs at 13:24 and 13:48 UT. This fast 19 May CME can be seen as a partial-halo event in STEREO/COR2 images, consistent with Earthward propagation. The EUVI $195 \AA$ movie (see supplementary online material: movie 3; http://dx.doi.org/10.1007/s11207-008-9300-y) shows that the flare and filament eruption were followed quickly by a dimming region at the flare site and the formation of a posteruption loop arcades at the flare site (seen in all EUVI channels), both of which commonly accompany CMEs (Tripathi, Bothmer, and Cremades, 2004). The arcade spans the southern end of the filament channel (see Figure 3).

The 20 May CME was associated with a B6.7-class flare also from AR 10956. The flare started at 04:52 UT and peaked at 05:56 UT. As for the 19 May event, a clear dimming region is seen at the flare site, as well as the formation of a post-eruptive arcade, both visible in the EUVI $195 \AA$ A movie (see supplementary online material: movie 4; http://dx.doi.org/10.1007/s11207-008-9300-y). The ejection of filament material can be clearly seen in the SECCHI/EUVI $304 \AA$ movies between 04:30 and 06:00 UT. The CME was first seen in LASCO C2 at 06:48 UT and can be seen in the SECCHI/COR2 movie as a partial-halo CME, consistent with Earthward propagation.

Next we compare the orientation of the magnetic cloud's axis to the orientation of the associated solar features. Figure 11 shows the smoothed magnetogram with contours (left panel, from Li et al., 2008) and an illustrative schematic of the neutral lines in AR 10956 (right panel). As shown in Figure 11b, both the 19 May and 20 May CMEs originated from the same complex neutral line that doubles back on itself, giving rise to a multipolar configuration. By using the Li et al. (2008) notation, two distinct sections of the complex neutral line have been identified: The West Neutral Line (WNL) is associated with the 19 May CME and MC1, and the Center Neutral Line (CNL) is associated with the 20 May CME and MC2. These sections of the neutral line are colored in pink, corresponding approximately to the spatial extent of the filament material observed to disappear in each case. 

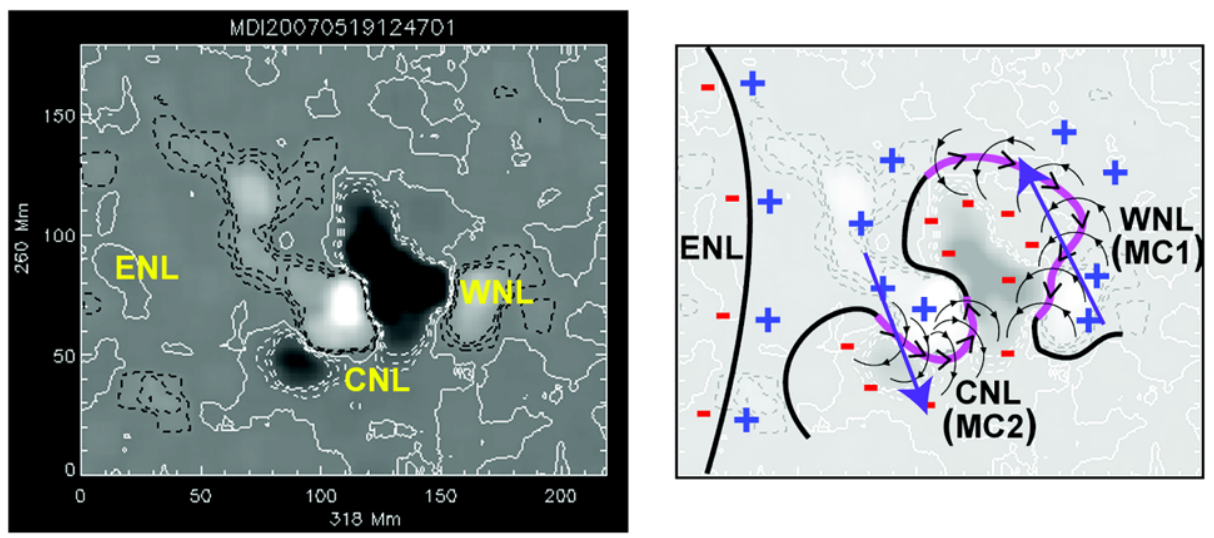

Figure 11 (Left) Smoothed magnetogram with contours. The solid white lines are magnetic neutral lines and the dashed black (white) lines are positive (negative) contours. WNL $=$ west neutral line; $\mathrm{CNL}=$ central neutral line; $\mathrm{ENL}=$ east neutral line (from Li et al., 2008). (Right) Illustrative schematic of AR 10956 and the associated CME source regions. Black thick lines show the neutral lines and the purple lines indicate the portions of the neutral line associated with filament material.

The streamer-belt neutral line when passing over AR 10956 (Figure 4) and the posteruptive arcades after the 19 May CME (Figure 3, white dashed line) had very similar orientations: Their orientation angles, determined with respect to the solar Equator, when measured from solar East (see Figure 3 and Yurchyshyn, 2008) were $\approx 140^{\circ}$. In comparison, the $\mathrm{MC} 1$ orientation angle, defined as the direction angle of the projected magnetic cloud axis onto the GSE $X Z$-plane and measured in the clockwise direction from the positive $Y$-axis (Yurchyshyn, 2008), was $63^{\circ}$ (indicated by a red line in Figure 3). MC2 had the orientation of about $68^{\circ}$ and its agreement with the orientation of the 20 May post-eruptive arcades is better: As seen from movie 4; http://dx.doi.org/10.1007/s11207-008-9300-y, the post-eruptive arcades associated with the 20 May CME were highly inclined to the solar Equator, roughly at $90^{\circ}$.

Although there is reasonable agreement in the projected in-situ orientations and the overall neutral-line configuration, once the chirality of the interplanetary field structures are taken into account the association becomes increasingly complicated. We have also marked in Figure 11b the in-situ orientation of the flux rope axes from the GS reconstructions of $\mathrm{MC} 1$ and $\mathrm{MC} 2$ as the purple arrows over their respective source regions. Since there were no vector magnetogram observations available within four days prior to the eruptions, making an independent determination of the source region chirality is difficult. We have assumed and depicted the direction of the sheared filament channel field such that the source region field structures have the same right-handed chirality as observed in both in-situ events. However, now the interplanetary flux rope and source-region arcade magnetic orientation, for MC1 in particular, differ significantly. If the hypothesized source-region association is correct and the chirality is conserved throughout the magnetic reconnection of the eruption processes (as expected, being related to magnetic helicity), then $\mathrm{MC} 1$ must undergo about $\approx 180^{\circ}$ rotation during the eruption and/or interplanetary propagation. The erupting filament material seen in the He 304 A movie of the 19 May CME does shows an apparent $\approx 90^{\circ}$ rotation from an approximately North - South to an East - West orientation while still in the EUVI field of view (see electronic attachment of Li et al., 2008). There have been other recent observations of similar rotational dynamics in the low corona (e.g., Green et al., 2007), so this may in fact resolve the apparent orientation discrepancies. 


\section{Discussion}

The investigated period was characterized by two high-speed solar wind streams and a magnetic sector boundary crossing from an away to a toward sector. Two magnetic clouds were observed between these high-speed streams. The STEREO satellites were separated by $\approx 9^{\circ}$ (few thousand $R_{\mathrm{E}}$ ) at this time and they observed clear differences in solar wind characteristics. Wind and STEREO B, separated by only $\approx 3^{\circ}$, also recorded differences in the structure of magnetic clouds they encountered.

Wind and STEREO B clearly encountered the same magnetic cloud ("MC1") on 21 23 May, as was demonstrated by generally similar behavior of magnetic field components during the passage of the cloud. However, the magnetic-field signatures were most pronounced at the location of STEREO B and the reconstruction of the magnetic cloud by the Grad-Shafranov technique confirmed that STEREO B crossed MC1 almost centrally whereas Wind traversed the cloud significantly further away, passing about $0.3 d_{\mathrm{CL}}$ from the axis.

The interaction of MC1 and the following complex high-speed stream distorted the trailing portion of the cloud and may explain the distortion of the field at Wind with respect to that seen at STEREO B. In addition, STEREO B observed an interplanetary shock in the latter half of the MC1, whereas Wind observed a more gradual pressure increase. The shock affected the magnetic-cloud parameters at the rear end, by heating the plasma and compressing the plasma and magnetic field. Internal shocks have been previously reported in $\approx 10 \%$ of the Wind magnetic clouds and it was suggested that they might be caused by the interaction with a magnetic cloud and a trailing high-speed stream (Collier, Lepping, and Berdichevsky, 2007).

It was concluded that STEREO A probably crossed only the flanks of MC1. In this case the East - West separation between the STEREO satellites gives a lower limit of the longitudinal scale size of MC1. The longitudinal separation of STEREO satellites at the time of the event $\left(9.1^{\circ}\right)$ corresponds to a longitudinal separation of $\approx 0.16 \mathrm{AU}$ and thus to a longitudinal width of $0.32 \mathrm{AU}$. This is almost twice the estimated scale size in the radial direction at STEREO B for MC1. The oblate cross sections of magnetic clouds have been suggested by several simulations and observational studies (Crooker and Intriligator, 1996; Mulligan and Russell, 2001; Riley et al., 2003; Liu et al., 2006). GSR analysis suggested that STEREO A was outside MC1. If STEREO A indeed encountered MC1 this event is an example of the limitations in the flux rope fitting models. As discussed by Riley et al. (2004) the GSR technique may not capture the true distortion of the flux rope, presumably since the method assumes that the structure is in approximate magnetostatic equilibrium.

STEREO A encountered a magnetic cloud on 23 May ("MC2"). The behavior of the magnetic-field components was clearly different from that during $\mathrm{MC} 1$, and the axis orientation deduced from GSR for MC2 was almost opposite that for MC1. As deduced from the directional properties of the electron heat flux data, $\mathrm{MC} 1$ was associated with predominantly closed field lines, but during the passage of MC2 heliospheric field lines were open. In addition, even though of the observing spacecraft STEREO A was located closest to the Sun, the front boundary of MC2 arrived about a day later at STEREO A than the MC1 arrived at Wind and STEREO B. It was thus concluded that MC2 and MC1 were not manifestations of the same magnetic cloud. Wind probably observed a short interval of MC2. The longitudinal separation between Wind and STEREO A was $6^{\circ}$, implying the longitudinal width for $\mathrm{MC} 2$ was at least $0.2 \mathrm{AU}$, again larger than the estimated scale size in the radial direction (0.14 AU). For MC2 the GSR technique suggested that Wind should be located inside MC2. A period of magnetic-field directional changes at Wind matched quite well to those predicted by GSR, but no other magnetic cloud signatures were present at Wind. 
As shown by the results of this study, large CMEs can frequently be part of the streamerbelt outflows and they can affect the manner in which the sector boundary crossings occur. At the locations of STEREO A and STEREO B, the true sector boundary (reversal of electron heat flux direction) was observed roughly at the same time, early on 24 May. However, the characteristics of the transition of the magnetic azimuth angle from the away to the toward sector was very different at these satellites. At STEREO B and Wind the magnetic azimuth angle fluctuated between an away and a toward sector for about a day after the end boundary of a small magnetic cloudlike region that followed MC1 before the electron heat flux reversed. At the location of STEREO A the transition was gradual and confined within a large-scale region of field rotation consisting of MC2 and the following region.

Multipoint detection of magnetic clouds by the STEREO satellites has been suggested to be possible up to STEREO separations of $\approx 50^{\circ}$ (Kaiser et al., 2007). At the time of the investigated events, the STEREO satellites were only about $9^{\circ}$ apart. In addition to the magnetic clouds studied in this paper, during the period when STEREO satellites were separated from $0.05^{\circ}$ (January 2007) to $50^{\circ}$ (April 2008) only two clear magnetic clouds (19 November 2007 and 29-30 December 2007) have been identified from the satellite data. STEREO B encountered both of these clouds, but STEREO A did not intercept either of them. Wind did not detect signatures of the December 2007 magnetic cloud, but it clearly observed the November 2007 magnetic cloud. However, the magnetic cloud looked remarkably different at Wind and STEREO B. This raises the question of whether the encountered magnetic clouds have been limited in longitudinal direction because they were observed during solar minimum and distorted heavily by the interaction with the high-speed streams or whether the extents of magnetic clouds vary considerably in all phases of the solar cycle. It should be noted, however, that both MC1 and MC2 were highly inclined with respect to the ecliptic plane.

The investigated events took place during solar activity minimum conditions. Even so, it was not a straightforward matter to relate the solar and in-situ observations. From an analysis of the solar observations, the LASCO CME catalog, and the clouds' speeds at the spacecraft, we found that MC1 was most likely associated with the solar eruptions (filament, flare, and CME) on 19 May at about 13 UT and MC2 was mostly likely associated with the solar eruptions (filament, flare, and CME) on 20 May at about 4 UT. These events originated from active region 10956, which consisted of a multipolar, nested flux system (Li et al., 2008). The 19 May fast CME appeared to be part of a complex eruption that included a slow CME observed very close in time and space to the fast CME. These CMEs were difficult to separate in coronagraph measurements and it is unclear whether they are part of the same eruption. In-situ observations by STEREO B and Wind also support the interpretation of a complex eruption: Immediately following the MC1 end boundary, a small magnetic cloudlike region with counterstreaming electrons was identified.

It is interesting to note that if only STEREO A in-situ observations had been available, the solar source for MC2 could have been erroneously interpreted to be the 19 May fast CME and the associated filament eruption. Even though the solar source regions of the CMEs on 19 May and 20 May were almost at the disk center, the $\mathrm{L}_{1}$ satellites (Wind and ACE) crossed both of the cloud's relatively far away from the axis. If in-situ observations by solely the $\mathrm{L}_{1}$ satellites would have been available MC2 would not have been identified at all and also the identification of $\mathrm{MC} 1$ would have been difficult. In addition, a small magnetic cloud region after MC1 was observed clearly only at STEREO B.

The orientation of the streamer-belt neutral line and the orientations of the filament axis and the post-eruptive arcades have been shown to correlate with the orientation of the magnetic cloud axes in interplanetary space (Mulligan, Russell, and Luhmann, 1998; 
Zhao and Hoeksema, 1998; Yurchyshyn, 2008). The results of GSR analysis suggest that both MC1 and MC2 were highly inclined. The streamer-belt neutral line and post-eruptive arcades were also highly inclined but tilted toward the West, the opposite direction to the magnetic cloud's axis. However, the agreement between the orientation of the 20 May posteruptive arcades and the MC2 axis was fairly good, the difference being about $20^{\circ}$.

Surprisingly, the investigated magnetic clouds had almost the same magnetic fluxes even though it seems clear that they cannot be part of the same large-scale structure. MC1 and $\mathrm{MC} 2$ originated from the same active region, and from the same neutral line that formed a complex looplike structure doubling back to itself. As determined from in-situ observations both magnetic clouds had the same right-handed chirality but their axial fields were oppositely directed, one with a northward- and the other with a southward-pointing axial field. This clearly presents a challenge for any viable space-weather forecasting (Harra et al., 2007). As no vector magnetograms were available during the time of the event, we assumed that the direction of the filament field matches that determined from in-situ observations. Especially for MC1, its axis orientation differed quite drastically from the associated neutral line orientation, implying significant rotation during the eruption and interplanetary propagation.

\section{Summary}

We have analyzed solar observations and in-situ measurements by the Wind and STEREO satellites during a complex chain of events on 19-23 May 2007. The results of this paper show how multispacecraft measurements present a significant advance when connecting solar and interplanetary observations and studying large-scale structure of magnetic clouds and solar wind structure in which they are embedded. Signatures of two CMEs, launched less than a day apart from the same active region, located almost at the disk center, could be identified in situ only because observations from multiple well-separated satellites were available. The flux rope fitting technique based on the Grad-Shafranov reconstruction was applied successfully for both magnetic clouds, but the technique did not capture the true distortion of the flux ropes.

Even though magnetic clouds originated from the same active region and had comparable magnetic fluxes and same chirality, their axial fields were oppositely directed. The identification of the magnetic clouds was complicated because of the strong interaction with the ambient solar-wind structure. Observations from several vantage points were also necessary to correctly connect the CMEs with their interplanetary counterparts. In addition, the results in this paper highlight the complexity of the observations at $1 \mathrm{AU}$ when they are collected by distant spacecraft. It was found that the streamer-belt extension near $1 \mathrm{AU}$ can be far from a simple structure, and it can vary significantly owing to the intrusions of large-scale CMEs even at relatively small longitudinal distances at the quietest time of the solar cycle. Furthermore, the results indicate that magnetic clouds can look remarkably different at multipoint sites even for small separation angles. This can be partly due to preference of smaller magnetic clouds during solar minimum that interact strongly with the ambient solar wind and partly due to high inclination of the investigated magnetic clouds.

Acknowledgements Work at the University of California, Berkeley, was supported from STEREO Grant No. NAS5-03131. C. Möstl acknowledges funding from the Steiermärkische Sparkasse and by the Austrian FWF project P20145-N16. C. Farrugia acknowledges NASA Grant Nos. NNX08AD11G and NNG06GD41G. Antoinette Galvin acknowledges NASA Contract No. NAS5-00132. The SECCHI data used here were produced by an international consortium of the NRL (USA), LMSAL (USA), NASA/GSFC (USA), 
RAL (UK), MPS (Germany), CSL (Belgium), IOTA (France), and IAS (France). The work of P.C. Liewer was performed at the Jet Propulsion Laboratory, California Institute of Technology, under a contract with NASA. This work utilizes data obtained by the Global Oscillation Network Group (GONG) Program, managed by the National Solar Observatory, which is operated by AURA, Inc., under a cooperative agreement with the National Science Foundation. The data were acquired by instruments operated by the Big Bear Solar Observatory, High Altitude Observatory, Learmonth Solar Observatory, Udaipur Solar Observatory, Instituto de Astrofísica de Canarias, and Cerro Tololo Interamerican Observatory.

Open Access This article is distributed under the terms of the Creative Commons Attribution Noncommercial License which permits any noncommercial use, distribution, and reproduction in any medium, provided the original author(s) and source are credited.

\section{References}

Cane, H.V., Richardson, I.G., St. Cyr, O.C.: 1998, The interplanetary events of January to May 1997, as inferred from energetic particle data, and their relationship with solar event. Geophys. Res. Lett. 25, 2571.

Burlaga, L.: 1988, Magnetic clouds and force-free fields with constant alpha. J. Geophys. Res. 93, 7217.

Burlaga, L.F., Sittler, E., Mariani, F., Schwenn, R.: 1981, Magnetic loop behind an interplanetary shock. J. Geophys. Res. 86, 6673.

Collier, M.R., Lepping, R.P., Berdichevsky, D.B.: 2007, A statistical study of interplanetary shocks and pressure pulses internal to magnetic clouds. J. Geophys. Res. 112, A06102. doi:10.1029/2006JA011714.

Crooker, N.U., Intriligator, D.S.: 1996, A magnetic cloud as a distended flux rope occlusion in the heliospheric current sheet. J. Geophys. Res. 101, 24343.

Crooker, N.U., Gosling, J.T., Kahler, S.W.: 1998, Magnetic clouds at sector boundaries. J. Geophys. Res. 103, 301.

Crooker, N.U., Siscoe, G.L., Shodan, S., Webb, D.F., Gosling, J.T., Smith, E.J.: 1993, Multiple heliospheric current sheets and coronal streamer belt dynamics. J. Geophys. Res. 98, 9371.

Fenrich, F.R., Luhmann, J.G.: 1998, Geomagnetic response to magnetic clouds of different polarity. Geophys. Res. Lett. 25, 2999.

Gopalswamy, N., Lara, A., Yashiro, S., Kaiser, M.L., Howard, R.A.: 2001, Predicting the 1-AU arrival times of coronal mass ejections. J. Geophys. Res. 106 (A12), 29207.

Gosling, J.T., Birn, J., Hesse, M.: 1995, Three-dimensional magnetic reconnection and the magnetic topology of coronal mass ejection events. Geophys. Res. Lett. 22, 22869.

Gosling, J., Skoug, J.R., Feldman, W.: 2001, Solar wind electron halo depletions at $90^{\circ}$ pitch angle. Geophys. Res. Lett. 28 (22), 4155.

Gosling, J.T., Borrini, G., Asbridge, J.R., Bame, S.J., Feldman, W.C., Hansen, R.T.: 1981, Coronal streamers in the solar wind at 1 AU. J. Geophys. Res. 86, 5438.

Green, L.M., Kliem, B, Török, T., van Driel-Gesztelyi, L., Attrill, G.D.R.: 2007, Transient coronal sigmoids and rotating erupting flux ropes. Solar Phys. 246, 365-391.

Harra, L.K., Crooker, N.U., Mandrini, C.H., van Driel-Gesztelyi, L., Dasso, S., Wang, J., Elliott, H., Attrill, G., Jackson, B.V., Bisi, M.M.: 2007, How does large flaring activity from the same active region produce oppositely directed magnetic clouds? Solar Phys. 244, 95.

Hu, Q., Sonnerup, B.U.O.: 2002, Reconstruction of magnetic clouds in the solar wind: Orientations and configurations. J. Geophys. Res. 107(A7). doi:10.1029/2001JA000293.

Hu, Q., Smith, C.W., Ness, N.F., Skoug, R.M.: 2004, Multiple flux rope magnetic ejecta in the solar wind. J. Geophys. Res. 109, 3102. doi:10.1029/2003JA010101.

Hundhausen, A.J.: 1993, The sizes and locations of coronal mass ejections: SMM observations from 1980 and 1984-1989. J. Geophys. Res. 98, 13177.

Kaiser, M., Kucera, T.A., Davila, J.M., St. Cyr, O.C., Guhathakurta, M., Christian, E.: 2007, The STEREO mission: An introduction. Space Sci. Rev. 136, 5. doi:10.1007/s11214-007-9277-0.

Leblanc, Y., Dulk, G.A., Vourlidas, A., Bougeret, J.-L.: 2001, Tracing shock waves from the corona to 1 AU. J. Geophys. Res. 106, 25301.

Lepping, R.P., Jones, J.A., Burlaga, L.F.: 1990, Magnetic field structure of interplanetary magnetic clouds at 1 AU. J. Geophys. Res. 95(A8), 11957.

Li, Y., Lynch, B.J., Stenborg, G., Luhmann, J.G., Huttunen, K.E.J., Welsch, B.T., Liewer, P.C., Vourlidas, A.: 2008, The solar magnetic field and coronal dynamics of the eruption on 2007 May 19. Astrophys. J. Lett. 681, L37. 
Liu, Y., Richardson, J.D., Belcher, J.W., Wang, C., Hu, Q., Kasper, J.C.: 2006, Constraints on the global structure of magnetic clouds: Transverse size and curvature. J. Geophys. Res. 111. doi:10.1029/2006JA011890.

Liu, Y., Luhmann, J.G., Huttunen, K.E.J., Lin, R.B., Bale, S.D., Russell, C.T., Galvin, A.B.: 2008, Reconstruction of the 2007 May 22 magnetic cloud: How much can we trust the flux-rope geometry of CMEs? Astrophys. J. Lett. 677, L133.

Mierla, M., Davila, J., Thompson, W., Inhester, B., Srivastava, N., Kramar, M., St. Cyr, O.C., Stenborg, G., Howard, R.A.: 2008, A quick method for estimating the propagation direction of coronal mass ejections using STEREO-COR1 images. Solar Phys. 252, 385-396.

Mulligan, T., Russell, C.T.: 2001, Multispacecraft modeling of the flux rope structure of interplanetary coronal mass ejections: Cylindrically symmetric versus nonsymmetric topologies. J. Geophys. Res. 106(A6), 10581.

Mulligan, T., Russell, C.T., Luhmann, J.G.: 1998, Solar cycle evolution of the structure of magnetic clouds in the inner heliosphere. Geophys. Res. Lett. 25, 2959.

Mulligan, T., Russell, C.T., Anderson, B.J., Lohr, D.A., Rust, D., Toth, B.A., Zanetti, L.J., Acuna, M.H., Lepping, R.P., Golsing, J.T.: 1999, Intercomparison of NEAR and Wind interplanetary coronal mass ejection observations. J. Geophys. Res. 104, 28217.

Neugebauer, M., Goldstein, R.: 1997, Particle and field signatures of coronal mass ejections in the solar wind. In: Crooker, N., Joselyn, J.A., Feynman, J. (eds.) Coronal Mass Ejections, Geophys. Monogr. 99, AGU, Washington, 245.

Plunkett, S.P., Thompson, B.J., Howard, R.A., Michels, D.J., St. Cyr, O.C., Tappin, S.J., Schwenn, R., Lamy, P.L.: 1998, LASCO observations of an Earth-directed coronal mass ejection on May 12, 1997. Geophys. Res. Lett. 25, 2477.

Qiu, J., Hu, Q., Howard, T.A., Yurchyshyn, V.B.: 2007, On the magnetic flux budget in low-corona magnetic reconnection and interplanetary coronal mass ejections. Astrophys. J. 659, 758. doi:10.1086/512060.

Riley, P., Linker, J.A., Mikic, Z., Odstrcil, D., Zurbuchen, T.H., Lario, D., Lepping, R.P.: 2003, Using an MHD simulation to interpret the global context of a coronal mass ejection observed by two spacecraft. J. Geophys. Res. 108 (A7), 1272.

Riley, P., Linker, J.A., Lionello, R., Mikic, Z., Odstrcil, D., Hidalgo, M.A., Cid, C., Hu, Q., Lepping, R.P., Lynch, B.J., Rees, A.: 2004, Fitting flux ropes to a global MHD solution: A comparison of techniques. J. Atmos. Solar Terr. Phys. 66, 1321.

Shodhan, S., Crooker, N.U., Kahler, W., Fitzenreiter, R.J., Larson, D.E., Lepping, R.P., Siscoe, G.L., Gosling, J.T.: 2000, Counterstreaming electrons in magnetic clouds. J. Geophys. Res. 105, 27261.

Tripathi, D., Bothmer, V., Cremades, H.: 2004, The basic characteristics of EUV post-eruptive arcades and their role as tracers of coronal mass ejections source regions. Astron. Astrophys. 422, 337. doi:10.1051/0004-6361:20035815.

Webb, D.F., Cliver, E.W., Crooker, N.U., St. Cyr, O.C., Thompson, B.J.: 2000, Relationship of halo coronal mass ejections, magnetic clouds, and magnetic storms. J. Geophys. Res. 105, 7491.

Yashiro, S., Gopalswamy, N., Michalek, G., St. Cyr, O.C., Plunkett, S.P., Rich, N.B., Howard, R.A.: 2004, A catalog of white light coronal mass ejections observed by the SOHO spacecraft. J. Geophys. Res. 109, A07105. doi:10.1029/2003JA010282.

Yurchyshyn, V.: 2008, Relationship between EIT posteruption arcades, coronal mass ejections, the coronal neutral line, and magnetic clouds. Astrophys. J. 675, L49.

Zhao, X.-P., Hoeksema, J.T.: 1998, Central axial field direction in magnetic clouds and its relation to southward interplanetary magnetic field events and dependence on disappearing solar filaments. J. Geophys. Res. 103, 2077. 